\title{
Exploitation of the Host Ubiquitin System: Means by Legionella pneumophila
}

\author{
Jingjing Luo', Lidong Wang ${ }^{1}$, Lei Song ${ }^{1 *}$ and Zhao-Qing Luo ${ }^{2 *}$ \\ ${ }^{1}$ Department of Respiratory Medicine, Center for Pathogen Biology and Infectious Diseases, Key Laboratory of Organ \\ Regeneration and Transplantation of the Ministry of Education, The First Hospital of Jilin University, Changchun, China, \\ ${ }^{2}$ Department of Biological Sciences, Purdue University, West Lafayette, IN, United States
}

\section{OPEN ACCESS}

Edited by:

Monica Cartelle Gestal, Louisiana State University Health Shreveport, United States

Reviewed by:

Yury Belyi,

N. F. Gamaleya Scientific Research Institute of Epidemiology and Microbiology (RAMS), Russia

Tomoe Kitao,

Gifu University, Japan

Stanimir Ivanov,

Louisiana State University Health

Shreveport, United States

Vincent Joseph Starai,

University of Georgia, United States

*Correspondence:

Lei Song

Isong@jlu.edu.cn

Zhao-Qing Luo

luoz@purdue.edu

Specialty section:

This article was submitted to Infectious Agents and Disease,

a section of the journal

Frontiers in Microbiology

Received: 06 October 2021 Accepted: 30 November 2021 Published: 22 December 2021

Citation:

Luo J, Wang L, Song L and Luo Z-Q (2021) Exploitation of the Host Ubiquitin System: Means by

Legionella pneumophila.

Front. Microbiol. 12:790442. do: $10.3389 /$ fmicb.2021.790442
Ubiquitination is a commonly used post-translational modification (PTM) in eukaryotic cells, which regulates a wide variety of cellular processes, such as differentiation, apoptosis, cell cycle, and immunity. Because of its essential role in immunity, the ubiquitin network is a common target of infectious agents, which have evolved various effective strategies to hijack and co-opt ubiquitin signaling for their benefit. The intracellular pathogen Legionella pneumophila represents one such example; it utilizes a large cohort of virulence factors called effectors to modulate diverse cellular processes, resulting in the formation a compartment called the Legionella-containing vacuole (LCV) that supports its replication. Many of these effectors function to re-orchestrate ubiquitin signaling with distinct biochemical activities. In this review, we highlight recent progress in the mechanism of action of $L$. pneumophila effectors involved in ubiquitination and discuss their roles in bacterial virulence and host cell biology.

Keywords: ubiquitin, intracellular pathogen, effector, immunity, virulence

\section{INTRODUCTION}

Ubiquitin (Ub) is a small, 76-amino acid protein that highly conserved in all eukaryotes (Liu et al., 2013; Shaid et al., 2013). The attachment of ubiquitin to client proteins forms a covalent modification known as ubiquitination, resulting in changes in the stability, cellular localization and/or activity of the substrates. Classical ubiquitination is a three-reaction cascade catalyzed by three types of enzymes (Figure 1). The first reaction is carried out by the ubiquitin-activating enzyme (E1), which catalyzes acyl adenylation on the carboxyl terminus of Ub by hydrolyzing an ATP molecule. The labile intermediate AMP-Ub then reacts with E1, resulting in linkage of the Ub moiety to its active cysteine residue by a thioester bond and the release of AMP (Hershko and Ciechanover, 1998). The Ub on E1 is then delivered to the active cysteine residue of ubiquitinconjugating enzyme (E2) (Olsen and Lima, 2013). Charged E2s carrying a Ub moiety work together with ubiquitin ligation enzymes (E3) to transfer the modifier onto a substrate (Buetow and Huang, 2016). Whereas E2s often dictate the chain type of ubiquitination (Stewart et al., 2016), E3s normally determine substrate specificity (Buetow and Huang, 2016). There are three major classes of E3 enzymes that utilize distinct mechanisms to transfer Ub from E2 or E3 itself to the substrate (Buetow and Huang, 2016). In most cases, Ub is attached to substrates by an isopeptide bond formed between its carboxyl terminus and the $\varepsilon$-amino group of Lys residues in target protein or in the preceding Ub moiety (Hershko and Ciechanover, 1998). 


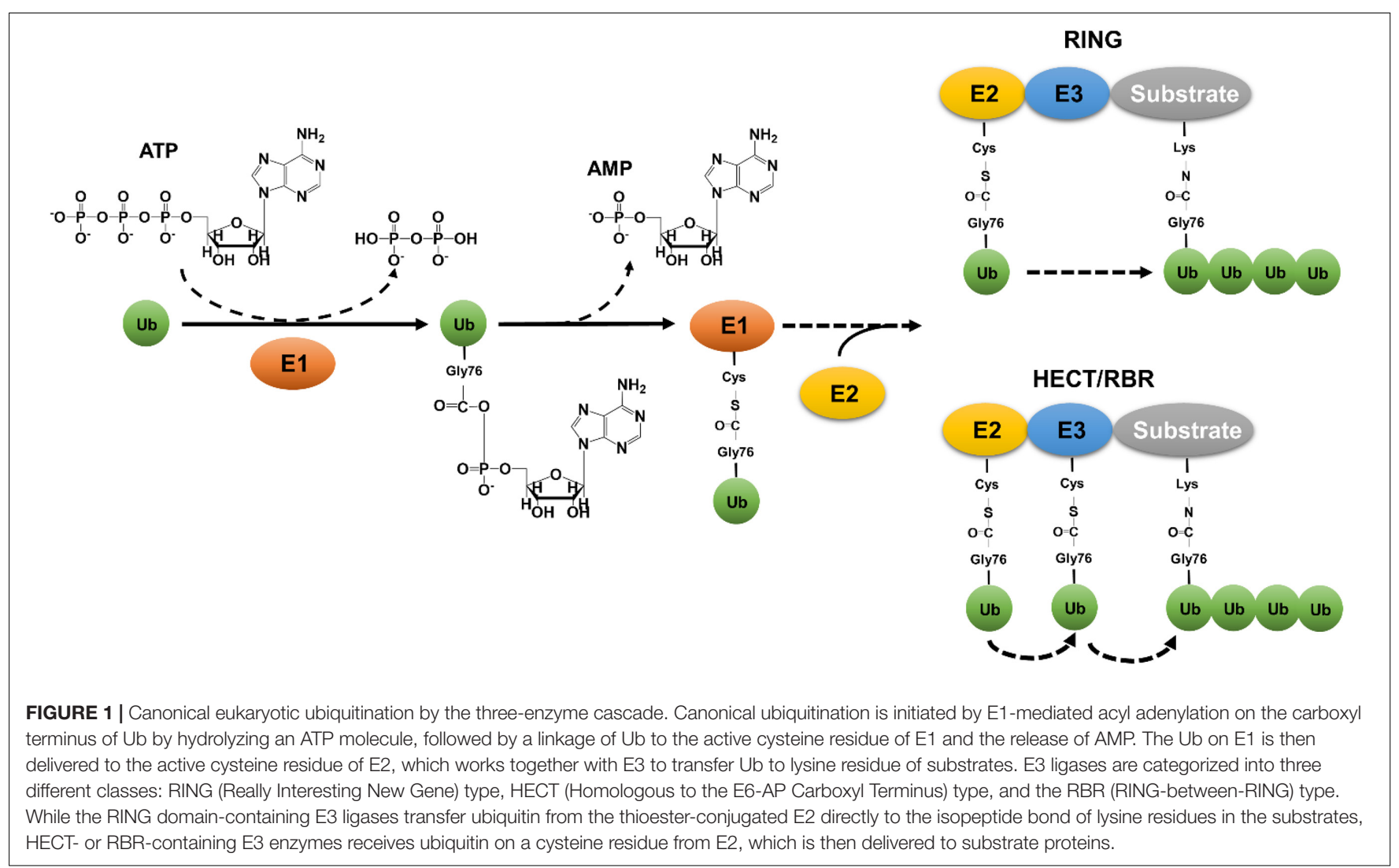

Ubiquitination can be reversed by deubiquitinases (Dubs), which are a large family of enzymes that cleave the isopeptide bond between $\mathrm{Ub}$ and its substrate or between $\mathrm{Ub}$ moieties, leading to the release of free $\mathrm{Ub}$ and the return of the substrate protein to its original state (Sowa et al., 2009). Together with E3 ligases, Dubs regulate a wide variety of cellular processes, such as apoptosis, cell cycle and division, mutation repair, transcription, differentiation, development, inflammation and immune responses (Hershko and Ciechanover, 1998). Under certain disease and development conditions, ubiquitin signaling is regulated by posttranslational modifications on one or more of the enzymes or Ub itself, which allow the cell to fine-tune specific signal transduction cascades regulated by ubiquitination (Song and Luo, 2019). Furthermore, a recent exciting discovery demonstrates that the lipid A moiety of bacterial lipopolysaccharide (LPS) is ubiquitinated by the E3 ubiquitin ligase RNF213, an event critical for the elimination of invading pathogenic Salmonella Typhimurium (Otten et al., 2021). The identification of non-proteinaceous substrates has greatly expanded the scope of the biological processes regulated by this posttranslational modification.

Because of the involvement of ubiquitination in diverse cellular processes, particularly in immunity, it is fulfilling to realize that many pathogens elaborate various pathogenic factors to co-opt ubiquitin signaling to facilitate their survival and replication in their hosts. Virulence factors involved in interference of the host ubiquitin network have been identified in a wide spectrum of pathogens (Zhou and Zhu, 2015;
Vozandychova et al., 2021). Among these, the opportunistic pathogen Legionella pneumophila is emerging as a unique model in this regard because of the sheer number of bacterial proteins involved and the richness in their mechanisms of action (Qiu and Luo, 2017b).

L. pneumophila is a Gram-negative bacterium ubiquitously found in fresh water environments where it exists as a parasite for protists. Inhalation of bacteria-contaminated aerosol by susceptible individuals can lead to the development of a severe form of pneumonia called Legionnaires' disease (Newton et al., 2010). L. pneumophila employs similar strategies to survive and replicate in amoebae and human macrophages, in both cases, it utilizes the Dot/Icm type IV secretion system to transport more than 330 effector proteins into host cells to construct the Legionella-containing vacuole (LCV) supportive for bacterial growth (Swanson and Isberg, 1995; Finsel and Hilbi, 2015; Ensminger, 2016). The LCV highly resembles the endoplasmic reticulum (ER) in term of protein composition and other cell biological features (Swanson and Isberg, 1995; Kagan and Roy, 2002). These Dot/Icm effectors interfere with a wide range of host processes with diverse biochemical activities (Qiu and Luo, 2017b). Among them, close to 20 effectors have been found to co-opt the host ubiquitin network by diverse biochemical mechanisms (Table 1; Qiu and Luo, 2017a). Here, we will highlight recent progress in the study of Legionella proteins that hijack host ubiquitination and their roles in bacterial pathogenesis and in the study of host cell biology. 
TABLE 1 | L. pneumophila Dot/lcm effectors involved in ubiquitination pathway.

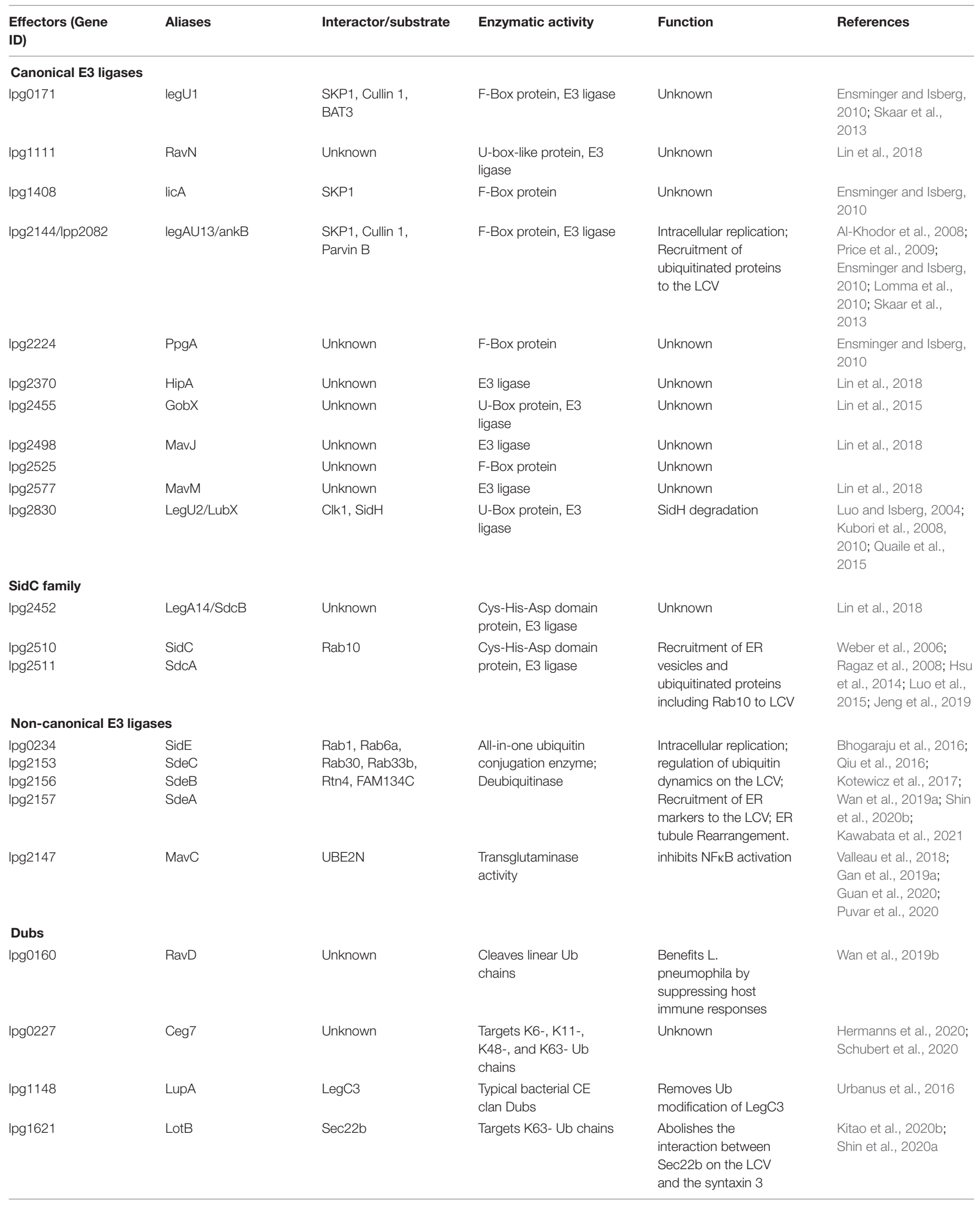


TABLE 1 | (Continued)

\begin{tabular}{|c|c|c|c|c|c|}
\hline $\begin{array}{l}\text { Effectors (Gene } \\
\text { ID) }\end{array}$ & Aliases & Interactor/substrate & Enzymatic activity & Function & References \\
\hline lpg2248 & LotA & Unknown & $\begin{array}{l}\text { Targets K13-, K48-, } \\
\text { and K63- Ub chains }\end{array}$ & $\begin{array}{l}\text { Removes ubiquitinated } \\
\text { proteins from the } \\
\text { surface of LCV }\end{array}$ & Kubori et al., 2018 \\
\hline lpg2529 & LotC/Lem27 & Rab10 & $\begin{array}{l}\text { Targets K6-, K11-, } \\
\text { K48-, and K63- Ub } \\
\text { chains }\end{array}$ & $\begin{array}{l}\text { Removes ubiquitinated } \\
\text { Rab10 from the surface } \\
\text { of LCV }\end{array}$ & $\begin{array}{l}\text { Liu et al., 2020; } \\
\text { Schubert et al., 2020; } \\
\text { Shin et al., 2020a }\end{array}$ \\
\hline
\end{tabular}

\section{CANONICAL E3 LIGASES}

The U-box type of E3s usually contain a conserved U-box sequence of 70 amino acids at their carboxyl termini (Miyamoto and Saito, 2018). LubX (Lpg2830) and GobX (Lpg2455) are L. pneumophila effectors that belong to this E3 family. Interestingly, LubX contains two U-box motifs with distinctly different functions: only U-box1 confers E3 activity (Quaile et al., 2015) and the proteins targeted by this enzyme appear structurally diverse. First, it attacks SidH (Lpg2829), a large Dot/Icm substrate (Luo and Isberg, 2004) encoded by a gene next to $l u b X$ by ubiquitination and subsequent degradation by the proteasome (Kubori et al., 2010). Because LubX functions to regulate the activity of another effector, it was designated as a metaeffector (Kubori et al., 2010). Interestingly, metaeffectors seem to be used in the regulation of a large cohort of Legionella effectors (Urbanus et al., 2016; Joseph and Shames, 2021; McCloskey et al., 2021). Second, LubX mediates ubiquitination of the Cdc2-like kinase 1 (CLK1) in host cells, leading to its degradation, but the biological significance of this degradation remains unknown (Kubori et al., 2008).

The effector GobX is the second U-box E3 ligase encoded by $L$. pneumophila, this enzyme is $S$-palmitoylated in host cells, thus allowing it to specifically target to the Golgi apparatus (Lin et al., 2015). Although its substrate has not yet been identified, such localization suggests that GobX functions to modulate cellular activity associated with this organelle (Lin et al., 2015). Recently, Lin et al. (2018) reported the amino terminal portion of RavN (Lpg1111) contains a U-box-like motif that has E3 activity. These authors also found that HipA (Lpg2370), MavM (Lpg2577), and Mavj (Lpg2498) have E3 ligase activity despite the fact that they share very limited homology to eukaryotic E3s. Unfortunately, the substrates of these E3s are currently unknown, so is their biological significance in L. pneumophila infection.

The SCF complex is a multi-subunit ubiquitin ligase consisting of RING-Box1 (RBXI), cullin 1 (CUL1), S-phase kinaseassociated protein 1 (SPK1) and an F-box motif containing protein (FBP) (Nguyen and Busino, 2020). Whereas the first three proteins serve as structural scaffold for the complex to interact with other proteins, particularly an appropriate E2 enzyme, FBP contributes to substrate specificity of the SCF complex by modules involved in protein-protein interaction such as leucine-rich repeats and WD repeats (Nguyen and Busino, 2020). The L. pneumophila strain Philadelphia 1 encodes at least 5 FBP-like effectors to engage ubiquitination (Ensminger and Isberg, 2010; Skaar et al., 2013). Among these, LegU1
(Lpg0171) and AnkB (Lpg2144) contain bona fide F-box motifs and have been demonstrated to form active SCF complexes by interacting with SKP1. LicA (Lpg1408) harbors an F-box motif and this protein interacts with SKP1 but does not detectably bind CUL1 (Ensminger and Isberg, 2010). The E3 ligase complex formed by LegU1 catalyzes ubiquitination of the HLAB-associated transcript-3 (BAT3) (Ensminger and Isberg, 2010), a protein involved in the regulation of a wide range of cellular processes, including apoptosis in higher eukaryotes (Sasaki et al., 2007; Corduan et al., 2009). By a yeast twohybrid screen, Lomma et al. (2010) identified ParvB as a substrate for AnkB and found that the bacterial F-box protein functions to reduce ParvB ubiquitination induced by endogenous enzymes. Yet whether ParvB is a specific substrate ubiquitylated by AnkB needs further study. AnkB is required for optimal intracellular growth of L. pneumophila strains AA100 and Paris in human monocyte derived macrophages (hMDMs) and for lung colonization in A/J mice (Price et al., 2009; Lomma et al., 2010). However, AnkB is dispensable for intracellular replication of strain Paris in Acanthamoeba castellanii (Lomma et al., 2010), while it is required for proficient replication of strain AA100 in A. polyphaga (Al-Khodor et al., 2008). These discrepancies may be due to differential effector redundancy in different strains of L. pneumophila or/and different protozoa infection models used. Finally, although both PpgA (Lpg2224) and Lpg2525 are predicted to harbor an F-box by bioinformatic analysis, neither has been shown to interact with SKP1 (Ensminger and Isberg, 2010). Whether these proteins have E3 ligase activity remained to be determined.

\section{SidC AND SdcA}

One prominent feature of the LCV is that this organelle dynamically recruits and retains an array of proteins of either host or L. pneumophila origin. In some cases, the anchoring of bacterial proteins on the LCV is mediated by their binding to phosphatidylinositol-4-phosphate (PI4P), an important signaling lipid whose enrichment on the bacterial phagosome is achieved in part by sequential action of three independent effectors that coordinate to biosynthesize PI4P from phosphatidylinositol (Hsu et al., 2012; Dong et al., 2016; Li et al., 2021). SidC (Lpg2511) and its ortholog SdcA anchor on the LCV by binding to PI4P via a lipid-interacting domain localized in the carboxyl portion of these proteins (Weber et al., 2006; Ragaz et al., 2008; Luo et al., 2015). These two highly similar effectors function to facilitate 
the recruitment of ER-derived vesicles and ubiquitylated proteins to the LCV, which is executed by an activity conferred by their amino terminal domain (Ragaz et al., 2008), a region later found to possess E3 ligase activity (Hsu et al., 2014). The biochemical basis of ubiquitination catalyzed by SidC and SdcA is unique, which requires a Cys-His-Asp catalytic triad (Hsu et al., 2014), which often is associated with proteases (Buller and Townsend, 2013). Although these two proteins are of $72 \%$ identical in their primary sequences, SidC prefers the $\mathrm{E} 2 \mathrm{UbcH} 7$ for its activity and predominantly catalyzes the formation of K11- and K33-linked polyubiquitin chains. In contrast, SdcA displays the highest activity when working together with the E2 UbcH5 (Hsu et al., 2014). The E3 ligase activity of SidC and SdcA is essential for their role in the recruitment of ER components to the LCV (Hsu et al., 2014; Luo et al., 2015). Intriguingly, structural analysis reveals that the four $\alpha$-helix bundles of the PI4P-binding motif blocks the catalytic site for the E3 activity embedded in the SNL (SidC N-terminal ubiquitin ligase) domain (Luo et al., 2015). In biochemical reactions, truncation mutants lacking the PI4Pbinding domain exhibits more robust E3 ligase activity than the full-length protein. Consistently, inclusion of $\mathrm{PI}(4) \mathrm{P}$ in reactions with full-length SidC enhances its E3 ligase activity (Luo et al., 2015). Thus, SidC and SdcA become more active in the presence of PI(4)P, which may allow them to selectively ubiquitinate their substrates on the surface of the LCV. Interestingly, Lpg2452 $(\mathrm{SdcB})$ also possess a domain structurally resembling the catalytic center of SidC important for its E3 ligase activity, thus had been included as a member of the SidC family (Lin et al., 2018).

The small GTPase Rab1 is monoubiquitinated in macrophages infected with wild-type L. pneumophila but not the $\Delta$ sidCsdcA mutant, suggesting that Rab1 is a substrate of these E3 enzymes (Horenkamp et al., 2014). However, ubiquitination of Rab1 by SidC or SdcA was not detected in cells co-expressing these proteins by transfection or in biochemical reactions with recombinant proteins (Horenkamp et al., 2014; Hsu et al., 2014). How infection of wild-type L. pneumophila induces Rab1 ubiquitination remains to be investigated. Recently, Jeng et al. (2019) identified the GTPase Rab10 as a substrate of SidC and SdcA. Consistent with the non-degradative K11- and K33-types of polyubiquitin chains induced by SidC and SdcA, Rab10 is not degraded by SidC or SdcA. Instead, ubiquitination leads to its recruitment to the LCV (Jeng et al., 2019). How ubiquitination of Rab10 and its recruitment to the bacterial phagosome benefit the bacterium is unknown.

\section{DUBS}

Ubiquitination can be reversed by enzymes of the deubiquitinase superfamily. Members of this large family of proteases function to cleave Ub from ubiquitinated substrates by hydrolyzing the isopeptide bond between $\mathrm{Ub}$ moieties and between $\mathrm{Ub}$ and the substrate (Harrigan et al., 2018). The specificity of Dubs toward their substrates is dictated by multiple factors, including their topography, the polyubiquitin chain types, and the way the enzyme makes contact with Ub moieties (Ronau et al., 2016). L. pneumophila codes for multiple Dubs that participate in various aspects of its interactions with host cells (Kitao et al., 2020a). Among these, LupA (Lpg1148) is the first described Dub in this pathogen, it harbors a Cys-His-Asp catalytic triad associated with typical bacterial CE clan Dubs (Urbanus et al., 2016). LupA is able to suppress the yeast toxicity of LegC3 (Lpg1701), another Dot/Icm substrate that appears to modulate membrane fusion in host cells (Urbanus et al., 2016). LegC3 undergoes ubiquitination in host cells probably by a yet unidentified host E3 ligase and LupA can remove such modification in a process that requires an intact Cys-His-Asp catalytic triad (Urbanus et al., 2016). How the Dub activity of LupA suppresses the yeast toxicity of LegC3 is not fully understood. One possibility is that ubiquitination activates the enzymatic activity of LegC3, which causes yeast toxicity and the removal of the Ub by LupA thus will put the toxicity under check.

LotA (Lpg2248) harbors two ovarian tumor (OTU)-like domains each possessing its own catalytic cysteine residue (Cys13 and Cys303, respectively). Whereas the domain using Cys13 mainly cleaves Lys6-linked polyubiquitin chains, the other prefers Lys48/Lys63-linked polyubiquitin (Kubori et al., 2018). Although its target is unknown, LotA contributes to the removal of ubiquitinated proteins from the surface of the LCV and is required for proficient intracellular growth of $L$. pneumophila (Kubori et al., 2018). LotB (Lpg1621) is another OTU-like Dub that specifically removes Lys63-linked ubiquitin chains (Ma et al., 2020; Schubert et al., 2020; Shin et al., 2020a). Structural analysis reveals that except for the ubiquitin binding site $S 1$, LotB harbors an additional site $\mathrm{S1}$, which confers its specificity in cleavage of the Lys63-linked chains (Shin et al., 2020a). The SNARE protein Sec22b involved in membrane fusion is a substrate of LotB; this protein is ubiquitinated in cells infected with L. pneumophila and LotB appears to function to counteract such modification (Shin et al., 2020a). The removal of ubiquitin from Sec22b abolishes the interaction between Sec22b on the LCV and the t-SNARE syntaxin 3, resulting in the dissociation of the latter from the bacterial phagosome (Kitao et al., 2020b). Interestingly, ubiquitination and recruitment of Rab10 to the LCV by SidC and SdcA are regulated by a third OUT-like Dub Lem27 (Lpg2529) (also known as LotC) coded for by L. pneumophila (Schubert et al., 2020; Shin et al., 2020a). Lem27 appears to modulate Rab10 ubiquitination by counteracting the activity of SidC and SdcA (Liu et al., 2020). Ceg7 (Lpg0227) has also been shown to possess Dub activity and belongs to the OUT-like family (Hermanns et al., 2020; Schubert et al., 2020). This enzyme broadly targets K6-, K11-, K48-, and K63-linked polyubiquitin chains in biochemical assays but the biological significance of this activity is unclear (Schubert et al., 2020). A recent structural study revealed that an extended helical lobe (EHL) domain commonly shared in LotA, LotB and Lem 27 is responsible for their Ub binding, defining the Lot-OTUs as a unique class of Dubs (Takekawa et al., 2021).

RavD (Lpg0160) is an effector that is associated with the LCV by binding to phosphatidylinositol-3-phosphate (PI3P) (Pike et al., 2019). Although no impact on bacterial intracellular growth, deletion of ravD led to a significant increase of late endosome/lysosome markers such as LAMP-1 on the LCV (Pike et al., 2019). A more recent study revealed that RavD is a Dub that 
specifically cleaves linear ubiquitin chains (Wan et al., 2019b), a unique ubiquitin chain important for signaling in innate and adaptive immunity, and inflammatory signaling (Rittinger and Ikeda, 2017). The removal of the linear ubiquitin chains on the LCV by RavD benefits L. pneumophila by suppressing host immune responses (Wan et al., 2019b). The activity of RavD suggests $L$. pneumophila may more extensively interact with higher order hosts, including mammals, because the ubiquitin ligase complex LUBAC responsible for the formation of linear ubiquitin chain is not present in protozoan (Spit et al., 2019).

\section{PHOSPHORIBOSYL UBIQUITINATION AND ITS REGULATION}

The SidE family contains SdeA, SdeB, SdeC, and SidE, which are four large proteins. Among these, SidE, SdeA, and SdeC are almost identical at the primary sequence level, only with a few conserved substitutions throughout the proteins of about 1,500 residues. SdeB (1926 aa) is considerably larger than the other three members of the family, it contains an extra region of about 400 aa in its carboxyl end (Luo and Isberg, 2004). Members of the SidE family (SidEs) are functionally redundant in L. pneumophila virulence (Bardill et al., 2005). Careful biochemical analysis reveals that these effectors represent a novel Ub ligase that catalyzes ubiquitination by a mechanism that is chemically distinct from the canonical three-enzyme cascade. First, Ub activation in reaction catalyzed by SidEs is energized by nicotinamide adenine dinucleotide (NAD), which is hydrolyzed by a mono-ADP-ribosyltransferase activity (mART) to modify Arg42 of Ub to produce ADP-ribosylated Ub (ADPRUb) (Qiu et al., 2016; Figure 2A). Whereas the biochemical basis of this catalysis is similar to classical mART often found in bacterial toxins (Mikolcevic et al., 2021), ADPR-Ub produced by this reaction is utilized by a phosphodiesterase (PDE) activity also embedded in each of SidE family member, which cleaves the phosphoanhydride bond between the two phosphate atoms, resulting in the transfer of phosphoribosyl $\mathrm{Ub}$ (PR-Ub) to serine residues on substrate proteins and the release of AMP (Bhogaraju et al., 2016; Kotewicz et al., 2017; Figure 2A).

Members of the SidE family appear to attack a large cohort of structurally diverse host proteins, including a number of Rab small GTPases and numerous ER resident proteins such as Rtn4 and FAM134C (Bhogaraju et al., 2016; Qiu et al., 2016; Kotewicz et al., 2017; Wan et al., 2019a; Shin et al., 2020b). Although many of these substrates are similarly attacked by each of these ligases (Bhogaraju et al., 2016; Qiu et al., 2016; Kotewicz et al., 2017; Wan et al., 2019a; Shin et al., 2020b), it is not clear whether any of these proteins specifically modifies one or more host proteins. Structural analysis suggests that a binding cleft in the PDE domain is responsible for substrate recognition which engages a sequence motif in which the target serine is in the vicinity of hydrophobic residues and is flanked by proline residues (Kalayil et al., 2018).

During L. pneumophila infection, ubiquitination of Rtn4 by SidEs drastically promotes structural transformation of ER tubules, probably by inducing Rtn 4 oligomerization, thus providing a scaffold for the formation of tubule matrix-like structures (Kotewicz et al., 2017). For small GTPases such as Rab33b, modification induced by SidEs leads to slight inhibition of its GTPase activity (Qiu et al., 2016). The modification and subsequent recruitment of other ER proteins such as FAM134C to the LCV may prevent the delivery of the bacterial phagosome to the autophagic pathway (Wan et al., 2019a; Shin et al., 2020b). Phosphoribosyl ubiquitination of Rab33b by SidEs recruits this GTPases to the LCV, where its associates with another small GTPase Rab6A, which interacts with the ER via direct interaction with SNAREs. Thus, one role of the SidE family is to promote the fusion between the LCV and the ER (Kawabata et al., 2021).

Members of SidEs family also have classical Dub activity, each conferred by a domain formed by the first 200 residues of the proteins (Sheedlo et al., 2015). The cleavage of isopeptide bond by these Dub domains is catalyzed by a Cys-His-Asp triad commonly found in the CE clan proteases (Sheedlo et al., 2015). This Dub module cleaves Lys11, Lys48, and Lys63linked polyubiquitin chains, with a distinct preference for Lys63 linkages (Sheedlo et al., 2015). The Dub activity of SidEs function to reduce the association of ubiquitinated proteins on the LCV, which may antagonize the recruitment of host autophagy machinery to the phagosome. In addition, the low chain type specificity of these Dub domains probably function to replenish the Ub pool proximal to the LCV to provide the reaction precursor for phosphoribosyl ubiquitination.

Similar to canonical ubiquitination, modification by SidEs is reversible. The phospho-ribose linkage between $\mathrm{Ub}$ and the substrate cannot be cleaved by canonical Dubs. By testing a number of Legionella proteins predicted to harbor a PDE domain potentially involved in cleaving phosphodiester bond, two independent studies identified DupA (Lpg2154) and DupB (Lpg2509) as enzymes that function to remove PR-Ub from substrates (Akturk et al., 2018; Wan et al., 2019a; Figure 2A). Intriguingly, the Dup family can hydrolyze ADPR-Ub to produce PR-Ub and AMP (Akturk et al., 2018; Wan et al., 2019a; Figure 2A).

Structural studies reveal that the distance between the mART domain of SidEs and the PDE domain is approximately $70 \AA$ (Akturk et al., 2018; Dong et al., 2018; Kalayil et al., 2018), suggesting the need of a mechanism to channel the reaction intermediate ADPR-Ub produced by the mART domain to the second reaction center. Because ADPR-Ub is highly toxic to eukaryotic cells by interfering with canonical ubiquitination (Bhogaraju et al., 2016), hydrolysis of this reaction intermediate by DupA and DupB may prevent poisoning of the host cell by ADPR-Ub that accidentally escapes from the mART catalytic pocket. Whether and how PR-Ub, which is also toxic to eukaryotic cells (Bhogaraju et al., 2016), is further reduced to phosphoribose and free Ub is unknown.

In addition to DupA and DupB, which mainly function to reverse ubiquitination induced by SidEs, the activity of this family of Ub ligases is further regulated by SidJ, one protein of a twomember family (Liu and Luo, 2007). SidJ had been reported to disperse SidEs from the LCV (Jeong et al., 2015) and can suppress the toxicity of SidEs to eukaryotic cells (Havey and Roy, 2015; Jeong et al., 2015). Of note is that the regulation of cellular 

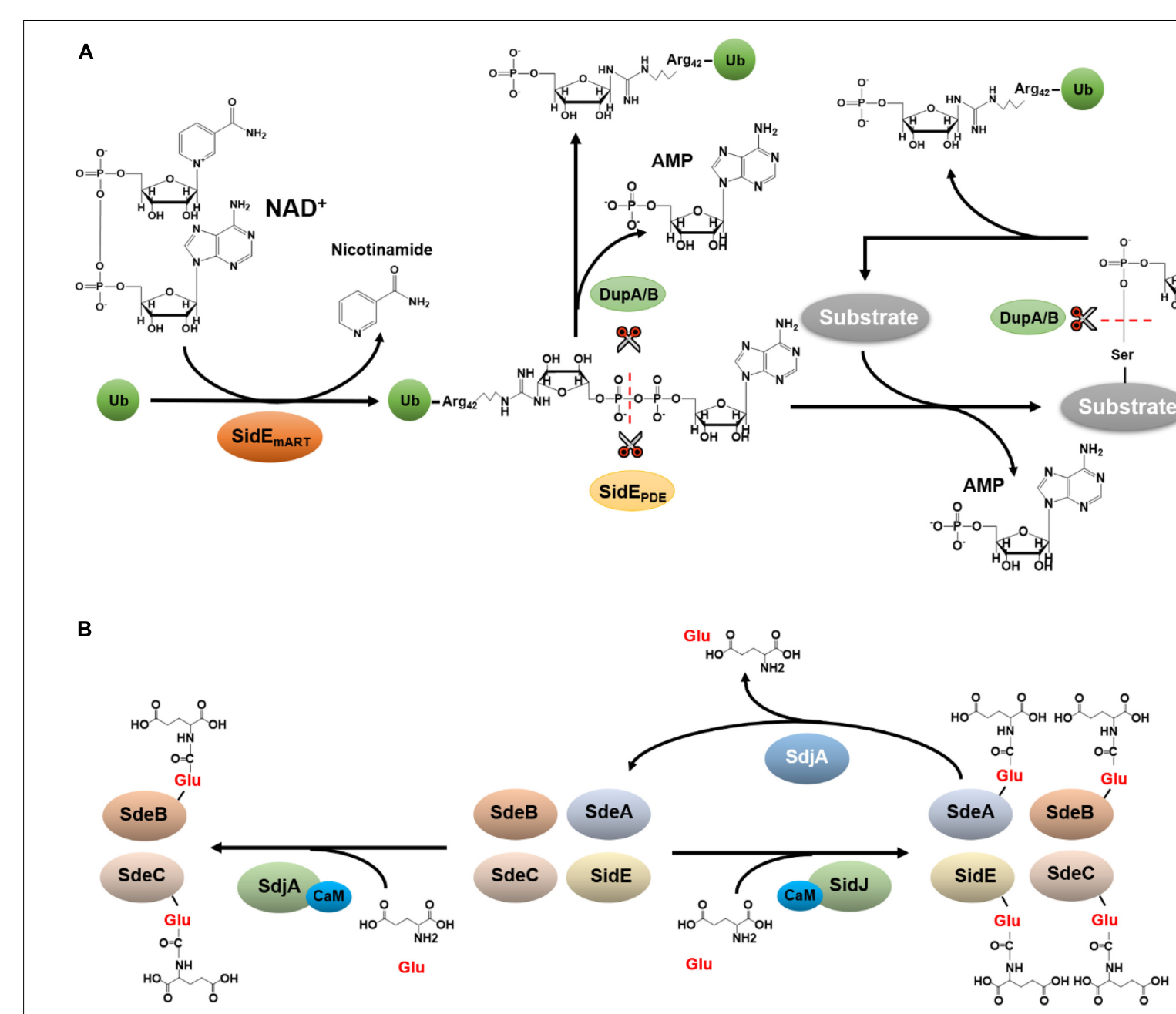

FIGURE 2 | Ubiquitination by the SidE family and its regulation. (A) Phosphoribosyl ubiquitination and de-ubiquitination. Phosphoribosyl ubiquitination induced by SidEs is initiated by ADP-ribosylation of Ub at Arg42 by its mART activity, leading to the release of nicotinamide and the production of ADPR-Ub. The phosphodiester bond in ADPR-Ub is subsequently the cleaved by the phosphodiesterase (PDE) domain of SidEs, which is accompanied by the transfer of phosphoribosyl Ub to serine residues of substrate proteins and the release of AMP. (B) Glutamylation and de-glutamylation of members of the SidE family. In host cells, SidJ binds to CaM via an IQ motif in its carboxyl end. The complex functions as a glutamylase to attack the first glutamate residue of the ExE motif in the mART domain of SidEs by polyglutamylation, leading to abolishment of the activity of the mART, and thus the inactivation of Ub ligase activity of the SidEs. Despite its high-level similarity to SidJ, SdjA selectively inhibits SdeB and SdeC by glutamylation. In addition to the glutamylase activity, SdjA also exhibits a deglutamylase activity toward glutamylated SdeA.

distribution of SidEs by SidJ was not detected in an independent study (Qiu et al., 2017). Four structural and biochemical studies independently reveal that SidJ is a glutamylase that attacks the first glutamate residue of the ExE motif in the mART domain of SidEs by polyglutamylation (Bhogaraju et al., 2019; Black et al., 2019; Gan et al., 2019b; Sulpizio et al., 2019). This modification abolishes the activity of the mART, thus the Ub ligase activity of the SidEs. Mechanistically, the glutamylation by SidJ is catalyzed by a two-step reaction in which the enzyme first activates the target glutamate residue by acyl-adenylation using a pseudokinase domain (Sreelatha et al., 2018), followed by the replacement of the AMP moiety with glutamate (Bhogaraju et al., 2019; Black et al., 2019; Gan et al., 2019b; Sulpizio et al., 2019), Interestingly, SidJ is activated by the eukaryote-specific calciumbinding protein calmodulin (CaM) which recognizes an IQ motif in its carboxyl end (Bhogaraju et al., 2019; Black et al., 2019; Gan et al., 2019b; Sulpizio et al., 2019). The requirement of CaM restricts the activity of SidJ in the cytosol of the infected cell, thus preventing premature inactivation of SidEs. Despite its highlevel similarity to SidJ, SdjA, the other member of the SidJ family, cannot rescue the yeast growth defect caused by SdeA (Qiu et al., 2017; Gan et al., 2019b). Furthermore, SidJ and SdjA are not functionally redundant for the intracellular growth defect of the $\Delta$ sidJ mutant cannot be complemented by SdjA (Liu and Luo, 2007). A recent study found that SdjA selectively inhibits some members of the SidE family by glutamylation (Osinski et al., 2021; Figure 2B). Interestingly, another study shows that in addition to the glutamylase activity against SdeB and SdeC, SdjA exhibits a deglutamylase activity toward SdeA that has been modified by SidJ/CaM (Song et al., 2021; Figure 2B). Thus, SdjA functions 


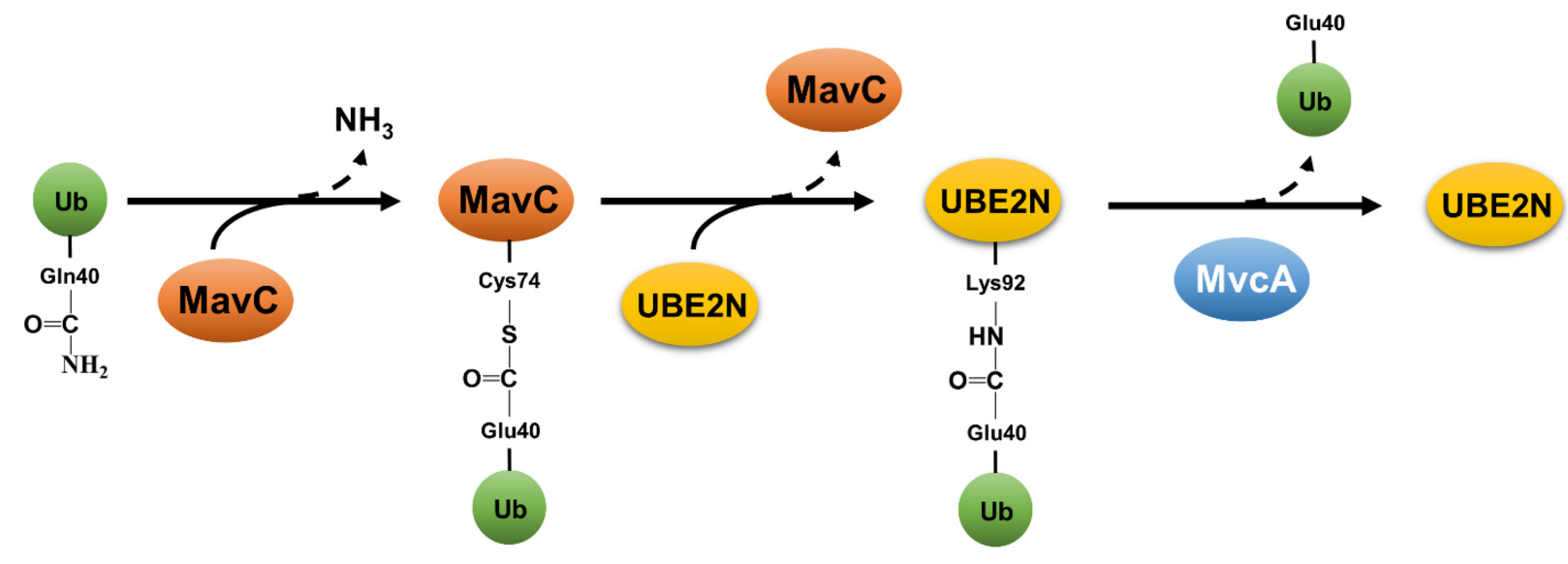

FIGURE 3 | Reversible atypical ubiquitination by MavC and MvcA. Ubiquitination catalyzed by MavC is initiated by the formation of a thioester linkage between the active residue Cys74 and Gln40 of Ub, concomitant with the release of ammonium. The acylated MavC then reacts with the amine donor from a lysine (Lys92) in UBE2N to form an intermolecular isopeptide bond, resulting in ubiquitination of UBE2N. MvcA, a structural ortholog of MavC, functions as a deubiquitinase to cleave the isopeptide bond between Ub and UBE2N catalyzed by MavC, leading to the release of UBE2N and deamidated Ub.

to fine-tune the activity of SidJ and SdeA during L. pneumophila infection (Song et al., 2021). Yet, the mechanism by which SdjA catalyzes the cleavage of the isopeptide bond between glutamate residues in glutamylated SdeA needs further investigation.

The SidE effector family has long known to be important for optimal virulence of L. pneumophila (Song et al., 2021), and targeting the unique ubiquitin ligase activity with compounds is an anti-virulence strategy to control L. pneumophila infection. Several stable $\mathrm{NAD}^{+}$analogs capable of inhibiting the activity of SdeC at IC $_{50}$ of $28-39 \mu \mathrm{M}$ have been developed (Madern et al., 2020). It remains unclear whether these compounds can block intracellular replication of the pathogen.

\section{REVERSIBLE UBIQUITINATION BY TRANSGLUTAMINASES MavC AND MvcA}

The second non-canonical ubiquitination mechanism by L. pneumophila is exemplified by MavC (Lpg2147) that catalyzes a crosslink reaction between $\mathrm{Ub}$ and the E2 enzyme UBE2N (Gan et al., 2019a). MavC is a transglutaminase that induces the formation of a $\gamma$-glutamyl- $\varepsilon$-Lys isopeptide between Gln 40 of Ub and Lys92 of UBE2N (Gan et al., 2019a). This reaction appears to preferably occur intramolecularly in charged UBE2N that carries a Ub moiety covalently linked to its active site Cys87 (Puvar et al., 2020). The reaction catalyzed by MavC is initiated by the formation of a thioester linkage between its active site Cys74 and the side chain amide of Gln40 of Ub, which is followed by a transglutamination reaction that leads to the installation of an isopeptide bond between Lys92 of UBE2N and Gln40 of Ub (Guan et al., 2020; Puvar et al., 2020; Figure 3). Because of its structural homology to bacterial deamidases Cif and CHBP that attack Ub and NEDD8 at Gln40 (Cui et al., 2010), MavC was identified in an earlier study as a Ub deamidase (Valleau et al., 2018). All described transglutaminases exhibit a protein deamidation activity in reactions lacking the crosslink target (Lorand and Graham, 2003). Consistent with this notion, MavC deamidates $\mathrm{Ub}$ at $\mathrm{Gln}_{40}$ in reactions containing only the enzyme and Ub (Valleau et al., 2018; Gan et al., 2019a). Importantly, the $\mathrm{Ub}$ deamidation activity is only detectable in biochemical reactions with large amounts of proteins but not in cells infected with wild-type L. pneumophila (Valleau et al., 2018; Gan et al., 2019a). Structurally, the main domain of MavC resembles the structures of Cif effectors (Valleau et al., 2018), the insertion domain is involved in engaging its substrate UBE2N (Guan et al., 2020).

MvcA (Lpg2148) is an ortholog of MavC, these two proteins share $50 \%$ identity and $65 \%$ similarity in their sequences and the structures of these two proteins are superimposable (Cui et al., 2010). Both MavC and MvcA deamidates Ub at Gln40, and this activity had been suggested to interfere with Ub signaling at different phases of L. pneumophila infection (Cui et al., 2010). Despite this high-level similarity, MvcA cannot catalyze protein crosslink between Ub and UBE2N or several other E2 enzymes (Gan et al., 2020). Further biochemical experiments reveal that MvcA functions as deubiquitinase that cleaves the isopeptide bond between $\mathrm{Ub}$ and $\mathrm{UBE} 2 \mathrm{~N}$ in the crosslink product formed by MavC (Gan et al., 2020; Mu et al., 2020; Figure 3). MavC and MvcA utilize the same biochemical mechanism to catalyze two reactions that have an opposite effect toward the activity of UBE2N (Gan et al., 2020; Mu et al., 2020). It has been proposed that the outcome of the reaction is determined by the binding affinity of the enzyme to $\mathrm{Ub}, \mathrm{UBE} 2 \mathrm{~N}$ and the product UBE2N-Ub (Guan et al., 2020). The surface charge of the Tail domain of MvcA is repulsive to $\mathrm{Ub}$, which together with the lower binding affinity for UBE2N favor the dissociation of Ub from $\mathrm{MvcA}$, thus resulting in the cleavage of the isopeptide bond (Guan et al., 2020).

A main function of UBE2N is to catalyze the formation of K63-type polyubiquitin chains, which are involved in the regulation of diverse cellular activities, including immunity by 
inducing the activation of the NFKB pathway (Hodge et al., 2016). MavC-induced ubiquitination UBE2N inserts the Ub moiety in the catalytic pocket of the E2 enzyme (Gan et al., 2020; Mu et al., 2020), which blocks the entry of activated Ub to its Cys87 active site. Although MavC can effectively induce crosslink between free $\mathrm{Ub}$ and $\mathrm{UBE} 2 \mathrm{~N}$, charged $\mathrm{UBE} 2 \mathrm{~N}$ carrying a Ub moiety on its catalytic site Cys87 via a thioester bond appears to be the prefer substrate (Puvar et al., 2020). This modification effectively inhibits NFKB activation by cues that initiate the signaling upstream of UBE2N (Valleau et al., 2018; Gan et al., 2019a). Like many Dot/Icm effectors, MavC expresses at high levels in bacteria grown to the post-exponential phase (Gan et al., 2019a), which facilitates the initial attack of the host defense upon contact. As the infection proceeds to later phases, the activity of $\mathrm{UBE} 2 \mathrm{~N}$ is restored by MvcA to accommodate the need of NFKB activation for maintaining critical cellular functions such as cell survival (Losick and Isberg, 2006). Consistent with this notion, the expression of MvcA does not become detectable several hours after bacterial uptake (Gan et al., 2020). Interestingly, Lpg2149 which is encoded by a gene in the mavC and $m v c A$ locus, blocks the activity of both MavC and MvcA by direct binding (Mu et al., 2020). The biological significance of Lpg2149 largely remains mysterious, but its presence further highlights the importance of fine-tuning the activity of UBE2N during L. pneumophila infection.

\section{CONCLUSION AND PERSPECTIVES}

The study of L. pneumophila effectors involved in ubiquitination has greatly expanded our appreciation of the exploitation of $\mathrm{Ub}$ signaling by a pathogen. It has also extended our understanding of the chemical basis of ubiquitination. A recent study shows that $\mathrm{Ub}$ signaling plays important roles beyond the remodeling of phagosomal membranes (Ong et al., 2021). Several host proteins involved in ubiquitination, particularly the E2 enzyme

\section{REFERENCES}

Akturk, A., Wasilko, D. J., Wu, X., Liu, Y., Zhang, Y., Qiu, J., et al. (2018). Mechanism of phosphoribosyl-ubiquitination mediated by a single Legionella effector. Nature 557, 729-733. doi: 10.1038/s41586-018-0147-6

Al-Khodor, S., Price, C. T., Habyarimana, F., Kalia, A., and Abu Kwaik, Y. (2008). A Dot/Icm-translocated ankyrin protein of Legionella pneumophila is required for intracellular proliferation within human macrophages and protozoa. Mol. Microbiol. 70, 908-923. doi: 10.1111/j.1365-2958.2008.06453.x

Bardill, J. P., Miller, J. L., and Vogel, J. P. (2005). IcmS-dependent translocation of SdeA into macrophages by the Legionella pneumophila type IV secretion system. Mol. Microbiol. 56, 90-103. doi: 10.1111/j.1365-2958.2005.04539.x

Bhogaraju, S., Bonn, F., Mukherjee, R., Adams, M., Pfleiderer, M. M., Galej, W. P., et al. (2019). Inhibition of bacterial ubiquitin ligases by SidJ-calmodulin catalysed glutamylation. Nature 572, 382-386. doi: 10.1038/s41586-0191440-8

Bhogaraju, S., Kalayil, S., Liu, Y., Bonn, F., Colby, T., Matic, I., et al. (2016). Phosphoribosylation of ubiquitin promotes serine ubiquitination and impairs conventional ubiquitination. Cell 167, 1636-1649 e13. doi: 10.1016/j.cell.2016. 11.019

Black, M. H., Osinski, A., Gradowski, M., Servage, K. A., Pawlowski, K., Tomchick, D. R., et al. (2019). Bacterial pseudokinase catalyzes protein polyglutamylation
UBE2E1, and the E3 Ub ligase, CUL7, are important not only for L. pneumophila intracellular replication but also for Dot/Icmmediated protein translocation (Ong et al., 2021). Clearly, the involvement of the Ub system in L. pneumophila pathogenesis is deeper and more extensive than we have appreciated. It will be of great interest to determine whether and how these host enzymes impact the functionality of the bacterial protein translocation machinery and its substrates. An equally important question is whether these enzymes are targeted by L. pneumophila effectors involved in Ub signaling.

With a few exceptions, the cellular targets of most of the E3 ligases or Dubs remain unknown. Clearly, the identification of their substrates is essential in our understanding of these effectors in the intracellular life cycle of L. pneumophila. Because the affinity between E3 ligases and their substrates often is too low to be harnessed for substrate identification (Nagy and Dikic, 2010), new technologies such as orthogonal Ub transfer (OUT) (Zhao et al., 2012), effective proximity labeling by the bacterial biotin ligase BirA (Branon et al., 2018) and substrate trapping by fusion proteins (O'Connor et al., 2015), will surely be useful in this endeavor. It is anticipated that more exciting discoveries will be made in years to come in our investigation of $\mathrm{Ub}$ signaling in L. pneumophila virulence.

\section{AUTHOR CONTRIBUTIONS}

JL and LW drafted the first version of the manuscript. LS and Z-QL revised the manuscript with input from all authors.

\section{FUNDING}

Work in our labs was supported by the Jilin Science and Technology Agency grant 20200403117SF (LS) and the National Institutes of Health grant R01AI127465 (Z-QL).

to inhibit the SidE-family ubiquitin ligases. Science 364, 787-792. doi: 10.1126/ science.aaw7446

Branon, T. C., Bosch, J. A., Sanchez, A. D., Udeshi, N. D., Svinkina, T., Carr, S. A., et al. (2018). Efficient proximity labeling in living cells and organisms with TurboID. Nat. Biotechnol. 36, 880-887. doi: 10.1038/nbt.4201

Buetow, L., and Huang, D. T. (2016). Structural insights into the catalysis and regulation of E3 ubiquitin ligases. Nat. Rev. Mol. Cell Biol. 17, 626-642. doi: 10.1038/nrm.2016.91

Buller, A. R., and Townsend, C. A. (2013). Intrinsic evolutionary constraints on protease structure, enzyme acylation, and the identity of the catalytic triad. Proc. Natl. Acad. Sci. U.S.A. 110, E653-E661. doi: 10.1073/pnas.122105 0110

Corduan, A., Lecomte, S., Martin, C., Michel, D., and Desmots, F. (2009). Sequential interplay between BAG6 and HSP70 upon heat shock. Cell Mol. Life Sci. 66, 1998-2004. doi: 10.1007/s00018-009-9198-z

Cui, J., Yao, Q., Li, S., Ding, X., Lu, Q., Mao, H., et al. (2010). Glutamine deamidation and dysfunction of ubiquitin/NEDD8 induced by a bacterial effector family. Science 329, 1215-1218. doi: 10.1126/science.1193844

Dong, N., Niu, M., Hu, L., Yao, Q., Zhou, R., and Shao, F. (2016). Modulation of membrane phosphoinositide dynamics by the phosphatidylinositide 4-kinase activity of the Legionella LepB effector. Nat. Microbiol. 2:16236. doi: 10.1038/ nmicrobiol.2016.236 
Dong, Y., Mu, Y., Xie, Y., Zhang, Y., Han, Y., Zhou, Y., et al. (2018). Structural basis of ubiquitin modification by the Legionella effector SdeA. Nature 557, 674-678. doi: 10.1038/s41586-018-0146-7

Ensminger, A. W. (2016). Legionella pneumophila, armed to the hilt: justifying the largest arsenal of effectors in the bacterial world. Curr. Opin. Microbiol. 29, 74-80. doi: 10.1016/j.mib.2015.11.002

Ensminger, A. W., and Isberg, R. R. (2010). E3 ubiquitin ligase activity and targeting of BAT3 by multiple Legionella pneumophila translocated substrates. Infect. Immun. 78, 3905-3919. doi: 10.1128/IAI.00344-10

Finsel, I., and Hilbi, H. (2015). Formation of a pathogen vacuole according to Legionella pneumophila: how to kill one bird with many stones. Cell Microbiol. 17, 935-950. doi: 10.1111/cmi.12450

Gan, N., Guan, H., Huang, Y., Yu, T., Fu, J., Nakayasu, E. S., et al. (2020). Legionella pneumophila regulates the activity of UBE2N by deamidase-mediated deubiquitination. EMBO J. 39:e102806. doi: 10.15252/embj.2019102806

Gan, N., Zhen, X., Liu, Y., Xu, X., He, C., Qiu, J., et al. (2019b). Regulation of phosphoribosyl ubiquitination by a calmodulin-dependent glutamylase. Nature 572, 387-391. doi: 10.1038/s41586-019-1439-1

Gan, N., Nakayasu, E. S., Hollenbeck, P. J., and Luo, Z. Q. (2019a). Legionella pneumophila inhibits immune signalling via MavC-mediated transglutaminaseinduced ubiquitination of UBE2N. Nat. Microbiol. 4, 134-143. doi: 10.1038/ s41564-018-0282-8

Guan, H., Fu, J., Yu, T., Wang, Z. X., Gan, N., Huang, Y., et al. (2020). Molecular basis of ubiquitination catalyzed by the bacterial transglutaminase MavC. $A d v$. Sci. 7:2000871. doi: 10.1002/advs.202000871

Harrigan, J. A., Jacq, X., Martin, N. M., and Jackson, S. P. (2018). Deubiquitylating enzymes and drug discovery: emerging opportunities. Nat. Rev. Drug Discov. 17, 57-78. doi: 10.1038/nrd.2017.152

Havey, J. C., and Roy, C. R. (2015). Toxicity and SidJ-mediated suppression of toxicity require distinct regions in the SidE family of Legionella pneumophila effectors. Infect. Immun. 83, 3506-3514. doi: 10.1128/IAI.00497-15

Hermanns, T., Woiwode, I., Guerreiro, R. F., Vogt, R., Lammers, M., and Hofmann, K. (2020). An evolutionary approach to systematic discovery of novel deubiquitinases, applied to Legionella. Life Sci. Alliance 3:e202000838. doi: $10.26508 /$ lsa.202000838

Hershko, A., and Ciechanover, A. (1998). The ubiquitin system. Annu. Rev. Biochem. 67, 425-479.

Hodge, C. D., Spyracopoulos, L., and Glover, J. N. (2016). Ubc13: the Lys63 ubiquitin chain building machine. Oncotarget 7, 64471-64504. doi: 10.18632/ oncotarget.10948

Horenkamp, F. A., Mukherjee, S., Alix, E., Schauder, C. M., Hubber, A. M., Roy, C. R., et al. (2014). Legionella pneumophila subversion of host vesicular transport by SidC effector proteins. Traffic 15, 488-499. doi: 10.1111/tra.12158

Hsu, F., Luo, X., Qiu, J., Teng, Y. B., Jin, J., Smolka, M. B., et al. (2014). The Legionella effector SidC defines a unique family of ubiquitin ligases important for bacterial phagosomal remodeling. Proc. Natl. Acad. Sci. U.S.A. 111, 1053810543. doi: 10.1073/pnas.1402605111

Hsu, F., Zhu, W., Brennan, L., Tao, L., Luo, Z. Q., and Mao, Y. (2012). Structural basis for substrate recognition by a unique Legionella phosphoinositide phosphatase. Proc. Natl. Acad. Sci. U.S.A. 109, 13567-13572. doi: 10.1073/pnas. 1207903109

Jeng, E. E., Bhadkamkar, V., Ibe, N. U., Gause, H., Jiang, L., Chan, J., et al. (2019). Systematic identification of host cell regulators of Legionella pneumophila pathogenesis using a genome-wide CRISPR screen. Cell Host Microbe 26, 551-563.e6. doi: 10.1016/j.chom.2019.08.017

Jeong, K. C., Sexton, J. A., and Vogel, J. P. (2015). Spatiotemporal regulation of a Legionella pneumophila T4SS substrate by the metaeffector SidJ. PLoS Pathog. 11:e1004695. doi: 10.1371/journal.ppat.1004695

Joseph, A. M., and Shames, S. R. (2021). Affecting the effectors: regulation of Legionella pneumophila effector function by metaeffectors. Pathogens 10:108. doi: 10.3390/pathogens10020108

Kagan, J. C., and Roy, C. R. (2002). Legionella phagosomes intercept vesicular traffic from endoplasmic reticulum exit sites. Nat. Cell Biol. 4, 945-954. doi: $10.1038 /$ ncb883

Kalayil, S., Bhogaraju, S., Bonn, F., Shin, D., Liu, Y., Gan, N., et al. (2018). Insights into catalysis and function of phosphoribosyl-linked serine ubiquitination. Nature 557, 734-738. doi: 10.1038/s41586-018-0145-8
Kawabata, M., Matsuo, H., Koito, T., Murata, M., Kubori, T., Nagai, H., et al. (2021). Legionella hijacks the host Golgi-to-ER retrograde pathway for the association of Legionella-containing vacuole with the ER. PLoS Pathog. 17:e1009437. doi: 10.1371/journal.ppat.100943

Kitao, T., Nagai, H., and Kubori, T. (2020a). Divergence of Legionella effectors reversing conventional and unconventional ubiquitination. Front. Cell Infect. Microbiol. 10:448. doi: 10.3389/fcimb.2020.00448

Kitao, T., Taguchi, K., Seto, S., Arasaki, K., Ando, H., Nagai, H., et al. (2020b). Legionella manipulates non-canonical SNARE pairing using a bacterial deubiquitinase. Cell Rep. 32:108107. doi: 10.1016/j.celrep.2020.108107

Kotewicz, K. M., Ramabhadran, V., Sjoblom, N., Vogel, J. P., Haenssler, E., Zhang, M., et al. (2017). A single Legionella effector catalyzes a multistep ubiquitination pathway to rearrange tubular endoplasmic reticulum for replication. Cell Host Microbe 21, 169-181. doi: 10.1016/j.chom.2016.12.007

Kubori, T., Hyakutake, A., and Nagai, H. (2008). Legionella translocates an E3 ubiquitin ligase that has multiple U-boxes with distinct functions. Mol. Microbiol. 67, 1307-1319. doi: 10.1111/j.1365-2958.2008.06124.x

Kubori, T., Kitao, T., Ando, H., and Nagai, H. (2018). LotA, a Legionella deubiquitinase, has dual catalytic activity and contributes to intracellular growth. Cell Microbiol. 20:e12840. doi: 10.1111/cmi.12840

Kubori, T., Shinzawa, N., Kanuka, H., and Nagai, H. (2010). Legionella metaeffector exploits host proteasome to temporally regulate cognate effector. PLoS Pathog 6:e1001216. doi: 10.1371/journal.ppat.1001216

Li, G., Liu, H., Luo, Z. Q., and Qiu, J. (2021). Modulation of phagosome phosphoinositide dynamics by a Legionella phosphoinositide 3-kinase. EMBO Rep. 22:e51163. doi: 10.15252/embr.202051163

Lin, Y. H., Doms, A. G., Cheng, E., Kim, B., Evans, T. R., and Machner, M. P. (2015). Host cell-catalyzed S-palmitoylation mediates golgi targeting of the legionella ubiquitin ligase GobX. J. Biol. Chem. 290, 25766-25781. doi: 10.1074/jbc.M115. 637397

Lin, Y. H., Lucas, M., Evans, T. R., Abascal-Palacios, G., Doms, A. G., Beauchene, N. A., et al. (2018). RavN is a member of a previously unrecognized group of Legionella pneumophila E3 ubiquitin ligases. PLoS Pathog. 14:e1006897. doi: 10.1371/journal.ppat.1006897

Liu, S., Luo, J., Zhen, X., Qiu, J., Ouyang, S., and Luo, Z. Q. (2020). Interplay between bacterial deubiquitinase and ubiquitin E3 ligase regulates ubiquitin dynamics on Legionella phagosomes. Elife 9:e58114. doi: 10.7554/eLife.58114

Liu, X., Wang, Q., Chen, W., and Wang, C. (2013). Dynamic regulation of innate immunity by ubiquitin and ubiquitin-like proteins. Cytokine Growth Factor Rev. 24, 559-570. doi: 10.1016/j.cytogfr.2013.07.002

Liu, Y., and Luo, Z. Q. (2007). The Legionella pneumophila effector SidJ is required for efficient recruitment of endoplasmic reticulum proteins to the bacterial phagosome. Infect. Immun. 75, 592-603. doi: 10.1128/IAI.01278-06

Lomma, M., Dervins-Ravault, D., Rolando, M., Nora, T., Newton, H. J., Sansom, F. M., et al. (2010). The Legionella pneumophila F-box protein Lpp2082 $(A n k B)$ modulates ubiquitination of the host protein parvin $\mathrm{B}$ and promotes intracellular replication. Cell Microbiol. 12, 1272-1291. doi: 10.1111/j.14625822.2010.01467.x

Lorand, L., and Graham, R. M. (2003). Transglutaminases: crosslinking enzymes with pleiotropic functions. Nat. Rev. Mol. Cell Biol. 4, 140-156. doi: 10.1038/ nrm 1014

Losick, V. P., and Isberg, R. R. (2006). NF-kappaB translocation prevents host cell death after low-dose challenge by Legionella pneumophila. J. Exp. Med. 203, 2177-2189. doi: 10.1084/jem.20060766

Luo, X., Wasilko, D. J., Liu, Y., Sun, J., Wu, X., Luo, Z. Q., et al. (2015). Structure of the Legionella virulence factor, SidC reveals a unique PI(4)P-specific binding domain essential for its targeting to the bacterial phagosome. PLoS Pathog. 11:e1004965. doi: 10.1371/journal.ppat.1004965

Luo, Z. Q., and Isberg, R. R. (2004). Multiple substrates of the Legionella pneumophila Dot/Icm system identified by interbacterial protein transfer. Proc. Natl. Acad. Sci. U.S.A. 101, 841-846. doi: 10.1073/pnas.0304916101

Ma, K., Zhen, X., Zhou, B., Gan, N., Cao, Y., Fan, C., et al. (2020). The bacterial deubiquitinase Ceg23 regulates the association of Lys-63-linked polyubiquitin molecules on the Legionella phagosome. J. Biol. Chem. 295, 1646-1657. doi: 10.1074/jbc.RA119.011758

Madern, J. M., Kim, R. Q., Misra, M., Dikic, I., Zhang, Y., Ovaa, H., et al. (2020). Synthesis of stable $\mathrm{NAD}(+)$ mimics as inhibitors for the Legionella pneumophila 
Phosphoribosyl ubiquitylating enzyme SdeC. Chembiochem 21, 2903-2907. doi: 10.1002/cbic.202000230

McCloskey, A., Perri, K., Chen, T., Han, A., and Luo, Z. Q. (2021). The metaeffector MesI regulates the activity of the Legionella effector SidI through direct proteinprotein interactions. Microbes Infect. 23:104794. doi: 10.1016/j.micinf.2021. 104794

Mikolcevic, P., Hlousek-Kasun, A., Ahel, I., and Mikoc, A. (2021). ADPribosylation systems in bacteria and viruses. Comput. Struct. Biotechnol. J. 19, 2366-2383. doi: 10.1016/j.csbj.2021.04.023

Miyamoto, K., and Saito, K. (2018). Concise machinery for monitoring ubiquitination activities using novel artificial RING fingers. Protein Sci. 27, 1354-1363. doi: 10.1002/pro.3427

Mu, Y., Wang, Y., Huang, Y., Li, D., Han, Y., Chang, M., et al. (2020). Structural insights into the mechanism and inhibition of transglutaminase-induced ubiquitination by the Legionella effector MavC. Nat. Commun. 11:1774. doi: 10.1038/s41467-020-15645-7

Nagy, V., and Dikic, I. (2010). Ubiquitin ligase complexes: from substrate selectivity to conjugational specificity. Biol. Chem. 391, 163-169. doi: 10.1515/bc.2010.021

Newton, H. J., Ang, D. K., Van Driel, I. R., and Hartland, E. L. (2010). Molecular pathogenesis of infections caused by Legionella pneumophila. Clin. Microbiol. Rev. 23, 274-298. doi: 10.1128/cmr.00052-09

Nguyen, K. M., and Busino, L. (2020). The biology of F-box proteins: the SCF Family of E3 ubiquitin Ligases. Adv. Exp. Med. Biol. 1217, 111-122. doi: 10. 1007/978-981-15-1025-0_8

O’Connor, H. F., Lyon, N., Leung, J. W., Agarwal, P., Swaim, C. D., Miller, K. M., et al. (2015). Ubiquitin-activated interaction traps (UBAITs) identify E3 ligase binding partners. EMBO Rep. 16, 1699-1712. doi: 10.15252/embr.201540620

Olsen, S. K., and Lima, C. D. (2013). Structure of a ubiquitin E1-E2 complex: insights to E1-E2 thioester transfer. Mol. Cell 49, 884-896. doi: 10.1016/j.molcel. 2013.01.013

Ong, S. Y., Schuelein, R., Wibawa, R. R., Thomas, D. W., Handoko, Y., Freytag, S., et al. (2021). Genome-wide genetic screen identifies host ubiquitination as important for Legionella pneumophila Dot/Icm effector translocation. Cell Microbiol. 23:e13368. doi: 10.1111/cmi.13368

Osinski, A., Black, M. H., Pawłowski, K., Chen, Z., Li, Y., and Tagliabracci, V. S. (2021). Structural and mechanistic basis for protein glutamylation by the kinase fold. Mol. Cell 81, 4527-4539.e8. doi: 10.1016/j.molcel.2021.08.007

Otten, E. G., Werner, E., Crespillo-Casado, A., Boyle, K. B., Dharamdasani, V., Pathe, C., et al. (2021). Ubiquitylation of lipopolysaccharide by RNF213 during bacterial infection. Nature 594, 111-116. doi: 10.1038/s41586-02103566-4

Pike, C. M., Boyer-Andersen, R., Kinch, L. N., Caplan, J. L., and Neunuebel, M. R. (2019). The Legionella effector RavD binds phosphatidylinositol-3-phosphate and helps suppress endolysosomal maturation of the Legionella-containing vacuole. J. Biol. Chem. 294, 6405-6415. doi: 10.1074/jbc.RA118.007086

Price, C. T., Al-Khodor, S., Al-Quadan, T., Santic, M., Habyarimana, F., Kalia, A., et al. (2009). Molecular mimicry by an F-box effector of Legionella pneumophila hijacks a conserved polyubiquitination machinery within macrophages and protozoa. PLoS Pathog. 5:e1000704. doi: 10.1371/journal.ppat.1000704

Puvar, K., Iyer, S., Fu, J., Kenny, S., Negron Teron, K. I., Luo, Z. Q., et al. (2020). Legionella effector MavC targets the Ube2 $\mathrm{N}^{\sim} \mathrm{Ub}$ conjugate for noncanonical ubiquitination. Nat. Commun. 11:2365. doi: 10.1038/s41467-020-16211-x

Qiu, J., and Luo, Z. Q. (2017b). Legionella and Coxiella effectors: strength in diversity and activity. Nat. Rev. Microbiol. 15, 591-605. doi: 10.1038/nrmicro. 2017.67

Qiu, J., and Luo, Z. Q. (2017a). Hijacking of the host ubiquitin network by Legionella pneumophila. Front. Cell Infect. Microbiol. 7:487. doi: 10.3389/fcimb. 2017.00487

Qiu, J., Sheedlo, M. J., Yu, K., Tan, Y., Nakayasu, E. S., Das, C., et al. (2016). Ubiquitination independent of E1 and E2 enzymes by bacterial effectors. Nature 533, 120-124. doi: 10.1038/nature17657

Qiu, J., Yu, K., Fei, X., Liu, Y., Nakayasu, E. S., Piehowski, P. D., et al. (2017). A unique deubiquitinase that deconjugates phosphoribosyl-linked protein ubiquitination. Cell Res. 27, 865-881. doi: 10.1038/cr.2017.66

Quaile, A. T., Urbanus, M. L., Stogios, P. J., Nocek, B., Skarina, T., Ensminger, A. W., et al. (2015). Molecular characterization of LubX: functional divergence of the U-box fold by Legionella pneumophila. Structure 23, 1459-1469. doi: $10.1016 /$ j.str.2015.05.020
Ragaz, C., Pietsch, H., Urwyler, S., Tiaden, A., Weber, S. S., and Hilbi, H. (2008). The Legionella pneumophila phosphatidylinositol-4 phosphate-binding type IV substrate SidC recruits endoplasmic reticulum vesicles to a replicationpermissive vacuole. Cell Microbiol. 10, 2416-2433. doi: 10.1111/j.1462-5822. 2008.01219.x

Rittinger, K., and Ikeda, F. (2017). Linear ubiquitin chains: enzymes, mechanisms and biology. Open Biol. 7:170026. doi: 10.1098/rsob.170026

Ronau, J. A., Beckmann, J. F., and Hochstrasser, M. (2016). Substrate specificity of the ubiquitin and Ubl proteases. Cell Res. 26, 441-456. doi: 10.1038/cr.2016.38

Sasaki, T., Gan, E. C., Wakeham, A., Kornbluth, S., Mak, T. W., and Okada, H. (2007). HLA-B-associated transcript 3 (Bat3)/Scythe is essential for p300mediated acetylation of p53. Genes Dev. 21, 848-861. doi: 10.1101/gad.1534107

Schubert, A. F., Nguyen, J. V., Franklin, T. G., Geurink, P. P., Roberts, C. G., Sanderson, D. J., et al. (2020). Identification and characterization of diverse OTU deubiquitinases in bacteria. EMBO J. 39:e105127. doi: 10.15252/embj. 2020105127

Shaid, S., Brandts, C. H., Serve, H., and Dikic, I. (2013). Ubiquitination and selective autophagy. Cell Death Differ. 20, 21-30. doi: 10.1038/cdd.2012.72

Sheedlo, M. J., Qiu, J., Tan, Y., Paul, L. N., Luo, Z. Q., and Das, C. (2015). Structural basis of substrate recognition by a bacterial deubiquitinase important for dynamics of phagosome ubiquitination. Proc. Natl. Acad. Sci. U.S.A. 112, 15090-15095. doi: 10.1073/pnas.1514568112

Shin, D., Bhattacharya, A., Cheng, Y. L., Alonso, M. C., Mehdipour, A. R., Van Der Heden Van Noort, G. J., et al. (2020a). Bacterial OTU deubiquitinases regulate substrate ubiquitination upon Legionella infection. Elife 9:e58277. doi: 10.7554/eLife. 58277

Shin, D., Mukherjee, R., Liu, Y., Gonzalez, A., Bonn, F., Liu, Y., et al. (2020b). Regulation of phosphoribosyl-linked serine ubiquitination by deubiquitinases DupA and DupB. Mol. Cell 77, 164-179.e6. doi: 10.1016/j.molcel.2019.10.019

Skaar, J. R., Pagan, J. K., and Pagano, M. (2013). Mechanisms and function of substrate recruitment by F-box proteins. Nat. Rev. Mol. Cell Biol. 14, 369-381. doi: $10.1038 / \mathrm{nrm} 3582$

Song, L., and Luo, Z. Q. (2019). Post-translational regulation of ubiquitin signaling. J. Cell Biol. 218, 1776-1786. doi: 10.1083/jcb.201902074

Song, L., Xie, Y., Li, C., Wang, L., He, C., Zhang, Y., et al. (2021). The Legionella effector SdjA Is a bifunctional enzyme that distinctly regulates Phosphoribosyl ubiquitination. mBio 12:e0231621. doi: 10.1128/mBio.02316-21

Sowa, M. E., Bennett, E. J., Gygi, S. P., and Harper, J. W. (2009). Defining the human deubiquitinating enzyme interaction landscape. Cell 138, 389-403. doi: 10.1016/j.cell.2009.04.042

Spit, M., Rieser, E., and Walczak, H. (2019). Linear ubiquitination at a glance. J. Cell Sci. 132:jcs208512. doi: 10.1242 /jcs. 208512

Sreelatha, A., Yee, S. S., Lopez, V. A., Park, B. C., Kinch, L. N., Pilch, S., et al. (2018). Protein AMPylation by an evolutionarily conserved Pseudokinase. Cell 175, 809-821.e19. doi: 10.1016/j.cell.2018.08.046

Stewart, M. D., Ritterhoff, T., Klevit, R. E., and Brzovic, P. S. (2016). E2 enzymes: more than just middle men. Cell Res. 26, 423-440. doi: 10.1038/cr.2016.35

Sulpizio, A., Minelli, M. E., Wan, M., Burrowes, P. D., Wu, X., Sanford, E. J., et al. (2019). Protein polyglutamylation catalyzed by the bacterial calmodulindependent pseudokinase SidJ. Elife 8:e51162. doi: 10.7554/eLife.51162

Swanson, M. S., and Isberg, R. R. (1995). Association of Legionella pneumophila with the macrophage endoplasmic reticulum. Infect. Immun. 63, 3609-3620. doi: 10.1128/iai.63.9.3609-3620.1995

Takekawa, N., Kubori, T., Iwai, T., Nagai, H., and Imada, K. (2021). Structural basis of ubiquitin recognition by a bacterial OTU deubiquitinase LotA. J. Bacteriol. JB0037621.

Urbanus, M. L., Quaile, A. T., Stogios, P. J., Morar, M., Rao, C., Di Leo, R., et al. (2016). Diverse mechanisms of metaeffector activity in an intracellular bacterial pathogen, Legionella pneumophila. Mol. Syst. Biol. 12:893. doi: 10.15252/msb. 20167381

Valleau, D., Quaile, A. T., Cui, H., Xu, X., Evdokimova, E., Chang, C., et al. (2018). Discovery of Ubiquitin deamidases in the pathogenic arsenal of Legionella pneumophila. Cell Rep. 23, 568-583. doi: 10.1016/j.celrep.2018.03.060

Vozandychova, V., Stojkova, P., Hercik, K., Rehulka, P., and Stulik, J. (2021). The Ubiquitination system within bacterial host-pathogen interactions. Microorganisms 9:638. doi: 10.3390/microorganisms9030638

Wan, M., Wang, X., Huang, C., Xu, D., Wang, Z., Zhou, Y., et al. (2019b). A bacterial effector deubiquitinase specifically hydrolyses linear ubiquitin chains 
to inhibit host inflammatory signalling. Nat. Microbiol. 4, 1282-1293. doi: 10. 1038/s41564-019-0454-1

Wan, M., Sulpizio, A. G., Akturk, A., Beck, W. H. J., Lanz, M., Faca, V. M., et al. (2019a). Deubiquitination of phosphoribosyl-ubiquitin conjugates by phosphodiesterase-domain-containing Legionella effectors. Proc. Natl. Acad. Sci. U.S.A. 116, 23518-23526. doi: 10.1073/pnas. 1916287116

Weber, S. S., Ragaz, C., Reus, K., Nyfeler, Y., and Hilbi, H. (2006). Legionella pneumophila exploits $\mathrm{PI}(4) \mathrm{P}$ to anchor secreted effector proteins to the replicative vacuole. PLoS Pathog. 2:e46. doi: 10.1371/journal.ppat.002 0046

Zhao, B., Bhuripanyo, K., Zhang, K., Kiyokawa, H., Schindelin, H., and Yin, J. (2012). Orthogonal ubiquitin transfer through engineered E1-E2 cascades for protein ubiquitination. Chem. Biol. 19, 1265-1277. doi: 10.1016/j.chembiol. 2012.07.023

Zhou, Y., and Zhu, Y. (2015). Diversity of bacterial manipulation of the host ubiquitin pathways. Cell Microbiol. 17, 26-34. doi: 10.1111/cmi.12384
Conflict of Interest: The authors declare that the research was conducted in the absence of any commercial or financial relationships that could be construed as a potential conflict of interest.

Publisher's Note: All claims expressed in this article are solely those of the authors and do not necessarily represent those of their affiliated organizations, or those of the publisher, the editors and the reviewers. Any product that may be evaluated in this article, or claim that may be made by its manufacturer, is not guaranteed or endorsed by the publisher.

Copyright (c) 2021 Luo, Wang, Song and Luo. This is an open-access article distributed under the terms of the Creative Commons Attribution License (CC BY). The use, distribution or reproduction in other forums is permitted, provided the original author(s) and the copyright owner(s) are credited and that the original publication in this journal is cited, in accordance with accepted academic practice. No use, distribution or reproduction is permitted which does not comply with these terms. 